\title{
Khan type fixed point theorems in a generalized metric space
}

\author{
H. Piria , S. Rahrovi ${ }^{a}$, P. Kumam ${ }^{b, c, *}$ \\ ${ }^{a}$ Department of Mathematics, University of Bonab, Bonab, 5551761167, Iran. \\ ${ }^{b}$ Theoretical and Computational Science Center (TaCS-Center) \& Department of Mathematics, Science Laboratory \\ Building, Faculty of Science, King Mongkuts University of Technology Thonburi (KMUTT), 126 Pracha Uthit Road, Bang \\ Mod, Thung Khru, Bangkok, 10140, Thailand. \\ ${ }^{c}$ Department of Medical Research, China Medical University Hospital, China Medical University, No. 91, Hsueh-Shih Road, \\ Taichung, 40402, Taiwan.
}

\begin{abstract}
Existence and uniqueness of fixed points are established for a mapping satisfying a new type of contractive condition involving a rational expression on a generalized metric space. Some main results by Ahmad et al. [J. Ahmad, M. Arshad, C. Vetro, Int. J. Anal., 2013 (2013), 6 pages] are extended and generalized, also several particular cases and an illustrative example are given. (C)2016 All rights reserved.
\end{abstract}

Keywords: Fixed point, metric space.

2010 MSC: 74H10, 54H25.

\section{Introduction and preliminaries}

In the mid-sixties ten, fixed points results dealing with general contractive conditions with rational expressions were appeared. One of the well-known works in this direction were established by Khan [4]. Fisher [3] gave a revised version of Khan as follows:

\footnotetext{
*Corresponding author

Email addresses: hossein_piri1979@yahoo.com (H. Piri), s.rahrovi@bonabu.ac.ir (S. Rahrovi), poom.kumam@mail.kmutt.ac.th (P. Kumam)
} 
Theorem $1.1([3])$. Let $(X, d)$ be a complete metric space and let $T: X \rightarrow X$ satisfies

$$
d(T x, T y) \leq \begin{cases}k \frac{d(x, T x) d(x, T y)+d(y, T y) d(y, T x)}{d(x, T y)+d(T x, y)}, & \text { if } d(x, T y)+d(T x, y) \neq 0 \\ 0, & \text { if } d(x, T y)+d(T x, y)=0\end{cases}
$$

where $k \in[0,1)$ and $x, y \in X$. Then $T$ has a unique fixed point $x^{*} \in X$. Moreover, for all $x \in X$, the sequence $\left\{T^{n} x\right\}$ converges to $x^{*}$.

Ahmad et al. [1] gave a new version of Theorem 1.1 in the setting of generalized metric spaces as follows:

Theorem 1.2. Let $(X, d)$ be a complete generalized metric space and let $T: X \rightarrow X$ be a self-mapping such that for all $x, y \in X$

$$
d(T x, T y) \leq \begin{cases}\delta d(x, y)+\zeta \frac{d(x, T x) d(x, T y)+d(y, T y) d(y, T x)}{d(x, T y)+d(T x, y)}, & \text { ifd } d(x, T y)+d(T x, y) \neq 0 \\ 0, & \text { ifd }(x, T y)+d(T x, y)=0\end{cases}
$$

for all $x, y \in X$ and $x \neq y$, and for some $\delta, \zeta \in[0,1)$ with $\delta+\zeta<1$. Then $T$ has a unique fixed point $x^{*} \in X$. Moreover, for all $x \in X$, the sequence $\left\{T^{n} x\right\}$ converges to $x^{*}$.

The aim of this paper is to give a new version of Theorem 1.1 in the setting of generalized metric spaces. The following definitions will be needed in the sequel.

Definition $1.3([2])$. Let $\mathrm{X}$ be a nonempty set and $d: X \times X \rightarrow \mathbb{R}^{+}$a mapping such that for all $x, y \in X$ and for all distinct points $u, v \in X$, which are different from $x$ and $y$, one has

$(G M 1) d(x, y)=0$ if and only if $x=y$;

$(G M 2) d(x, y)=d(y, x)$;

$(G M 3) d(x, z) \leq d(x, u)+d(u, v)+d(v, z)]$.

Then $d$ is called generalized metric and the pair $(X, d)$ is called generalized metric space (or shortly GMS).

For some examples about generalized metric space, we refer readers to [1, 5, 6].

Definition $1.4([2])$. Let (X, d) be a GMS, $\left\{x_{n}\right\}_{n=1}^{\infty}$ be a sequence in $\mathrm{X}$ and $x \in X$. Then

(i) We say that $\left\{x_{n}\right\}_{n=1}^{\infty}$ is GMS convergent to $x$ if and only if $d\left(x_{n}, x\right) \rightarrow 0$ as $n \rightarrow \infty$. We denote this by $x_{n} \rightarrow x$.

(ii) We say that $\left\{x_{n}\right\}_{n=1}^{\infty}$ is a GMS Cauchy sequence if and only if, for each $\epsilon>0$, there exists a natural number $n(\epsilon)$ such that $d\left(x_{n}, x_{m}\right)<\epsilon$ for all $n>m>n(\epsilon)$.

(iii) $(X, d)$ is called GMS complete if every GMS Cauchy sequence is GMS convergent in $X$.

\section{Main results}

Theorem 2.1. Let $(X, d)$ be a complete GMS and let $T: X \rightarrow X$ be a self-mapping such that

$$
d(T x, T y) \leq\left\{\begin{array}{lc}
\gamma \max \left\{d(x, y), \frac{d(x, T x) d(x, T y)+d(y, T y) d(y, T x)}{\max \{d(x, T y), d(T x, y)\}},\right. & \text { if } \max \{d(x, T y), d(T x, y)\} \neq 0, \\
0, & \text { if } \max \{d(x, T y), d(T x, y)\}=0
\end{array}\right.
$$


for all $x, y \in X$ and $x \neq y$, and for some $\gamma \in[0,1)$. Then $T$ has a unique fixed point $x^{*} \in X$. Moreover, for all $x \in X$, the sequence $\left\{T^{n} x\right\}$ converges to $x^{*}$.

Proof. Let $x_{0}=x \in X$. Put $x_{n+1}=T x_{n}=T^{n+1} x_{0}$ for all $n \in \mathbb{N}_{0}=\mathbb{N} \cup\{0\}$. If, there exists $n \in \mathbb{N}$ such that $x_{n}=x_{n-1}$, then $x_{n-1}$ is a fixed point of $\mathrm{T}$. This completes the proof. Therefore, we suppose $x_{n} \neq x_{n-1}$ for all $n \in \mathbb{N}$. We shall divide the proof into two cases.

Cases 1. Assume that

$$
\max \left\{d\left(x_{m}, T x_{n}\right), d\left(T x_{m}, x_{n}\right)\right\} \neq 0
$$

for all $m \in \mathbb{N}$ and $n \in \mathbb{N}_{0}$. Then from (2.1), we have

$$
\begin{aligned}
d\left(x_{n}, x_{n+1}\right) & =d\left(T x_{n-1}, T x_{n}\right) \\
& \leq \gamma \max \left\{d\left(x_{n-1}, x_{n}\right), \frac{d\left(x_{n-1}, T x_{n-1}\right) d\left(x_{n-1}, T x_{n}\right)+d\left(x_{n}, T x_{n}\right) d\left(x_{n}, T x_{n-1}\right)}{\max \left\{d\left(x_{n-1}, T x_{n}\right), d\left(T x_{n-1}, x_{n}\right)\right\}}\right\} \\
& =\gamma \max \left\{d\left(x_{n-1}, x_{n}\right), \frac{d\left(x_{n-1}, x_{n}\right) d\left(x_{n-1}, x_{n+1}\right)+d\left(x_{n}, x_{n+1}\right) d\left(x_{n}, x_{n}\right)}{\max \left\{d\left(x_{n-1}, x_{n+1}\right), d\left(x_{n}, x_{n}\right)\right\}}\right\} \\
& =\gamma d\left(x_{n-1}, x_{n}\right) .
\end{aligned}
$$

Hence the sequence $\left\{d\left(x_{n}, x_{n+1}\right)\right\}$ is monotonic nonincreasing and bounded below. So, there exists $\zeta \geq 0$ such that

$$
\lim _{n \rightarrow \infty} d\left(x_{n}, x_{n+1}\right)=\zeta=\lim _{n \rightarrow \infty} d\left(x_{n-1}, x_{n}\right) .
$$

We claim that $\zeta=0$. Suppose by the contrary that $\zeta>0$. Taking limits as $n \rightarrow \infty$ to each side of the (2.2), we get $\zeta \leq \gamma \zeta<\zeta$. It is a contradiction. Therefore, we have

$$
\lim _{n \rightarrow \infty} d\left(x_{n}, T x_{n}\right)=\lim _{n \rightarrow \infty} d\left(x_{n}, x_{n+1}\right)=0 .
$$

A gain using inequality (2.1), we have

$$
\begin{aligned}
d\left(x_{n},\right. & \left.x_{n+2}\right) \\
& =d\left(T x_{n-1}, T x_{n+1}\right) \\
& \leq \gamma \max \left\{d\left(x_{n-1}, x_{n+1}\right), \frac{d\left(x_{n-1}, T x_{n-1}\right) d\left(x_{n-1}, T x_{n+1}\right)+d\left(x_{n+1}, T x_{n+1}\right) d\left(x_{n+1}, T x_{n-1}\right)}{\max \left\{d\left(x_{n-1}, T x_{n+1}\right), d\left(T x_{n-1}, x_{n+1}\right)\right\}}\right\} \\
& =\gamma \max \left\{d\left(x_{n-1}, x_{n+1}\right), \frac{d\left(x_{n-1}, x_{n}\right) d\left(x_{n-1}, x_{n+2}\right)+d\left(x_{n+1}, x_{n+2}\right) d\left(x_{n+1}, x_{n}\right)}{\max \left\{d\left(x_{n-1}, x_{n+2}\right), d\left(x_{n}, x_{n+1}\right)\right\}}\right\} \\
& \leq \gamma \max \left\{d\left(x_{n-1}, x_{n+1}\right), \frac{d\left(x_{n-1}, x_{n}\right) d\left(x_{n-1}, x_{n+2}\right)}{d\left(x_{n-1}, x_{n+2}\right)}+\frac{d\left(x_{n+1}, x_{n+2}\right) d\left(x_{n+1}, x_{n}\right)}{d\left(x_{n}, x_{n+1}\right)}\right\} \\
& =\gamma \max \left\{d\left(x_{n-1}, x_{n+1}\right), d\left(x_{n-1}, x_{n}\right)+d\left(x_{n+1}, x_{n+2}\right)\right\} \\
& \leq \gamma \max \left\{d\left(x_{n-1}, x_{n}\right)+d\left(x_{n}, x_{n+2}\right)+d\left(x_{n+2}, x_{n+1}\right), d\left(x_{n-1}, x_{n}\right)+d\left(x_{n+1}, x_{n+2}\right)\right\} \\
& \left.=\gamma\left[d\left(x_{n-1}, x_{n}\right)+d\left(x_{n}, x_{n+2}\right)+d\left(x_{n+2}, x_{n+1}\right)\right)\right] .
\end{aligned}
$$

It follows that

$$
d\left(x_{n}, x_{n+2}\right) \leq \frac{1}{1-\gamma}\left[d\left(x_{n-1}, x_{n}\right)+d\left(x_{n+2}, x_{n+1}\right)\right]
$$


So from (2.4), we get

$$
\lim _{n \rightarrow \infty} d\left(x_{n}, x_{n+2}\right)=0 .
$$

Now, we claim that, $\left\{x_{n}\right\}_{n=1}^{\infty}$ is a Cauchy sequence. Arguing by contradiction, we assume that there exist $\epsilon>0$, the sequences $\{p(n)\}_{n=1}^{\infty}$ and $\{q(n)\}_{n=1}^{\infty}$ of natural numbers such that

$$
p(n)>q(n)>n, \quad d\left(x_{p(n)}, x_{q(n)}\right) \geq \epsilon, d\left(x_{p(n)-1}, x_{q(n)}\right)<\epsilon, \quad \forall n \in \mathbb{N} .
$$

Observe that

$$
\begin{aligned}
\epsilon \leq d\left(x_{p(n)}, x_{q(n)}\right) & \leq d\left(x_{p(n)}, x_{p(n)-2}\right)+d\left(x_{p(n)-2}, x_{p(n)-1}\right)+d\left(x_{p(n)-1}, x_{q(n)}\right) \\
& =d\left(x_{p(n)}, x_{p(n)-2}\right)+d\left(x_{p(n)-2}, x_{p(n)-1}\right)+\epsilon .
\end{aligned}
$$

It follows from $(2.4)$ and 2.5 that

$$
\lim _{n \rightarrow \infty} d\left(x_{p(n)}, x_{q(n)}\right)=\epsilon
$$

From (2.1), we have

$$
\begin{aligned}
\epsilon & \leq d\left(x_{p(n)+1}, x_{q(n)+1}\right) \\
& \left.=d\left(T x_{p(n)}, T x_{q(n)}\right)\right) \\
& \leq \gamma \max \left\{d\left(x_{p(n)}, x_{q(n)}\right), \frac{d\left(x_{p(n)}, T x_{p(n)}\right) d\left(x_{p(n)}, T x_{q(n)}\right)+d\left(x_{q(n)}, T x_{q(n)}\right) d\left(x_{q(n)}, T x_{p(n)}\right)}{\max \left\{d\left(x_{p(n)}, T x_{q(n)}\right), d\left(T x_{p(n)}, x_{q(n)}\right)\right\}}\right\} \\
& =\gamma \max \left\{d\left(x_{p(n)}, x_{q(n)}\right), \frac{d\left(x_{p(n)}, x_{p(n)+1}\right) d\left(x_{p(n)}, x_{q(n)+1}\right)+d\left(x_{q(n)}, x_{q(n)+1}\right) d\left(x_{q(n)}, x_{p(n)+1}\right)}{\max \left\{d\left(x_{p(n)}, x_{q(n)+1}\right), d\left(x_{p(n)+1}, x_{q(n)}\right)\right\}}\right\} \\
& \leq \gamma \max \left\{d\left(x_{p(n)}, x_{q(n)}\right), \frac{d\left(x_{p(n)}, x_{p(n)+1}\right) d\left(x_{p(n)}, x_{q(n)+1}\right)}{d\left(x_{p(n)}, x_{q(n)+1}\right)}+\frac{d\left(x_{q(n)}, x_{q(n)+1}\right) d\left(x_{q(n)}, x_{p(n)+1}\right)}{d\left(x_{p(n)+1}, x_{q(n)}\right)}\right\} \\
& =\gamma \max \left\{d\left(x_{p(n)}, x_{q(n)}\right), d\left(x_{p(n)}, x_{p(n)+1}\right)+d\left(x_{q(n)}, x_{q(n)+1}\right)\right\} .
\end{aligned}
$$

It follows from (2.4) and (2.7) that $\epsilon \leq \gamma \epsilon<\epsilon$. This contradiction shows that $\left\{x_{n}\right\}$ is a cauchy sequence. By Completeness of $(X, d),\left\{x_{n}\right\}$ converges to some point $x^{*}$ in $X$. Therefore

$$
\lim _{n \rightarrow \infty} d\left(x_{n}, x^{*}\right)=0 .
$$

Observe that

$$
\begin{aligned}
d\left(x^{*}, T x^{*}\right) & \leq d\left(x^{*}, x_{n+1}\right)+d\left(x_{n+1}, x_{n}\right)+d\left(x_{n}, T x^{*}\right) \\
& \leq d\left(x^{*}, x_{n+1}\right)+d\left(x_{n+1}, x_{n}\right)+d\left(x_{n}, x_{n+1}\right)+d\left(x_{n+1}, x^{*}\right)+d\left(x^{*}, T x^{*}\right) \\
& \leq 2 d\left(x^{*}, x_{n+1}\right)+2 d\left(x_{n+1}, x_{n}\right)+d\left(x^{*}, T x^{*}\right) .
\end{aligned}
$$

It follows from (2.4) and (2.8) that

$$
\lim _{n \rightarrow \infty} d\left(x_{n}, T x^{*}\right)=d\left(x^{*}, T x^{*}\right) .
$$

On the other hand, from 2.1), we get

$$
\begin{aligned}
d\left(x_{n+1}, T x^{*}\right) & =d\left(T x_{n}, T x^{*}\right) \\
& \leq \gamma \max \left\{d\left(x_{n}, x^{*}\right), \frac{d\left(x_{n}, T x_{n}\right) d\left(x_{n}, T x^{*}\right)+d\left(x^{*}, T x^{*}\right) d\left(x^{*}, T x_{n}\right)}{\max \left\{d\left(x_{n}, T x^{*}\right), d\left(T x_{n}, x^{*}\right)\right\}}\right\} \\
& \leq \gamma \max \left\{d\left(x_{n}, x^{*}\right), \frac{d\left(x_{n}, x_{n+1}\right) d\left(x_{n}, T x^{*}\right)+d\left(x^{*}, T x^{*}\right) d\left(x^{*}, x_{n+1}\right)}{\max \left\{d\left(x_{n}, T x^{*}\right), d\left(x_{n+1}, x^{*}\right)\right\}}\right\} .
\end{aligned}
$$


So from (2.4), (2.8), 2.9) and taking limits as $n \rightarrow \infty$ to each side of (2.10), we have $d\left(x^{*}, T x^{*}\right)=0$. Now, we show that $T$ has a unique fixed point. For this, we assume that $y^{*}$ is another fixed point of $T$ in $X$ such that $d\left(x^{*}, y^{*}\right)>0$. Therefore

$$
\max \left\{d\left(x^{*}, T y^{*}\right), d\left(T x^{*}, y^{*}\right)\right\}=d\left(x^{*}, y^{*}\right)>0 .
$$

So from (2.1), we get

$$
\begin{aligned}
d\left(x^{*}, y^{*}\right) & =d\left(T x^{*}, T y^{*}\right) \\
& \leq \gamma \max \left\{d\left(x^{*}, y^{*}\right), \frac{d\left(x^{*}, T x^{*}\right) d\left(x^{*}, T y^{*}\right)+d\left(y^{*}, T y^{*}\right) d\left(y^{*}, T x^{*}\right)}{\max \left\{d\left(x^{*}, T y^{*}\right), d\left(T x^{*}, y^{*}\right)\right\}}\right\} \\
& \leq \gamma \max \left\{d\left(x^{*}, y^{*}\right), \frac{d\left(x^{*}, x^{*}\right) d\left(x^{*}, y^{*}\right)+d\left(y^{*}, y^{*}\right) d\left(y^{*}, x^{*}\right)}{\max \left\{d\left(x^{*}, y^{*}\right), d\left(x^{*}, y^{*}\right)\right\}}\right\} \\
& =\gamma d\left(x^{*}, y^{*}\right) .
\end{aligned}
$$

Since $\gamma \in[0,1)$. This leads to a contradiction and hence $x^{*}=y^{*}$. This complete the proof.

Cases 2. Assume that there exists $m \in \mathbb{N}$ and $n \in \mathbb{N}_{0}$ such that

$$
\max \left\{d\left(x_{m}, T x_{n}\right), d\left(T x_{m}, x_{n}\right)\right\}=0 .
$$

By condition (2.1), it follows that $d\left(T x_{m}, T x_{n}\right)=0$ and hence $x_{n}=T x_{m}=T x_{n}=x_{m}$. This completes the proof of the existence of a fixed point of $T$. The uniqueness follows as in Case 1.

Theorem 2.2. Let $(X, d)$ be a complete GMS and let $T: X \rightarrow X$ be a self-mapping such that for all $x, y \in X$

$$
d(T x, T y) \leq \begin{cases}\delta d(x, y)+\zeta \frac{d(x, T x) d(x, T y)+d(y, T y) d(y, T x)}{\max \{d(x, T y), d(T x, y)\}}, & \text { if } \max \{d(x, T y), d(T x, y)\} \neq 0 \\ 0, & \text { if } \max \{d(x, T y), d(T x, y)\}=0\end{cases}
$$

for all $x, y \in X$ and $x \neq y$, and for some $\delta, \zeta \in[0,1)$ such that $\delta+\zeta<1$. Then $T$ has a unique fixed point $x^{*} \in X$. Moreover, for all $x \in X$, the sequence $\left\{T^{n} x\right\}$ converges to $x^{*}$.

Proof. Since

$$
\begin{aligned}
\delta d(x, y)+ & \zeta \frac{d(x, T x) d(x, T y)+d(y, T y) d(y, T x)}{\max \{d(x, T y), d(T x, y)\}} \\
& \leq(\delta+\zeta) \max \left\{d(x, y), \frac{d(x, T x) d(x, T y)+d(y, T y) d(y, T x)}{\max \{d(x, T y), d(T x, y)\}}\right\} .
\end{aligned}
$$

So by taking $\gamma=\delta+\zeta$ in Theorem 2.1, the proof is complete.

Theorem 2.3 ([1]). Let $(X, d)$ be a complete generalized metric space and let $T: X \rightarrow X$ be a self-mapping such that for all $x, y \in X$

$$
d(T x, T y) \leq \begin{cases}\delta d(x, y)+\zeta \frac{d(x, T x) d(x, T y)+d(y, T y) d(y, T x)}{d(x, T y)+d(T x, y)}, & \text { ifd }(x, T y)+d(T x, y) \neq 0 \\ 0, & \text { ifd }(x, T y)+d(T x, y)=0\end{cases}
$$

for all $x, y \in X$ and $x \neq y$, and for some $\delta, \zeta \in[0,1)$ with $\delta+\zeta<1$. Then $T$ has a unique fixed point $x^{*} \in X$. Moreover, for all $x \in X$, the sequence $\left\{T^{n} x\right\}$ converges to $x^{*}$. 
Proof. Since

$$
\frac{d(x, T x) d(x, T y)+d(y, T y) d(y, T x)}{d(x, T y)+d(T x, y)} \leq \frac{d(x, T x) d(x, T y)+d(y, T y) d(y, T x)}{\max \{d(x, T y), d(T x, y)\}} .
$$

So from Theorem 2.2, the proof is complete.

Example 2.4. Let $X=\{0,1,2,3\}$ and define $d: X \times X \rightarrow \mathbb{R}$ as follows:

$$
\begin{aligned}
& d(0,0)=d(1,1)=d(2,2)=d(3,3)=0 \\
& d(0,3)=d(3,0)=d(2,3)=d(3,2)=d(1,2)=d(2,1)=1, \\
& d(0,2)=d(2,0)=d(1,3)=d(3,1)=2.1 \\
& d(0,1)=d(1,0)=1.1
\end{aligned}
$$

Then, $(X, d)$ is a complete GMS. Let $T: X \rightarrow X$ be defined by

$$
T x= \begin{cases}0, & \text { if } x \neq 3 \\ 1, & \text { if } x=3 .\end{cases}
$$

Now we consider the following cases:

Case1. Let $(x, y) \in\{(0,1),(1,0)\}$, then

$$
\begin{aligned}
& d(T 0, T 1)=0, d(0,1)=1.1, d(0, T 1)+d(T 0,1)=1.1 \\
& d(0, T 0) d(0, T 1)+d(1, T 1) d(1, T 0)=1.21, \max \{d(0, T 1), d(T 0,1)\}=1.1
\end{aligned}
$$

Case2. Let $(x, y) \in\{(0,2),(2,0)\}$, then

$$
\begin{aligned}
& d(T 0, T 2)=0, d(0,2)=2.1, d(0, T 2)+d(T 0,2)=2.1 \\
& d(0, T 0) d(0, T 2)+d(2, T 2) d(2, T 0)=4.4, \max \{d(0, T 2), d(T 0,2)\}=2.1 .
\end{aligned}
$$

Case3. Let $(x, y) \in\{(0,3),(3,0)\}$, then

$$
\begin{aligned}
& d(T 0, T 3)=1.1, d(0,3)=1, d(0, T 3)+d(T 0,3)=2.1 \\
& d(0, T 0) d(0, T 3)+d(3, T 3) d(3, T 0)=2.1, \max \{d(0, T 3), d(T 0,3)\}=1.1 .
\end{aligned}
$$

Case4. Let $(x, y) \in\{(1,2),(2,1)\}$, then

$$
\begin{aligned}
& d(T 1, T 2)=0, d(1,2)=1, d(1, T 2)+d(T 1,2)=3.1 \\
& d(1, T 1) d(1, T 2)+d(2, T 2) d(2, T 1)=5.62, \max \{d(1, T 2), d(T 1,2)\}=2.1
\end{aligned}
$$

Case5. Let $(x, y) \in\{(1,3),(3,1)\}$, then

$$
\begin{aligned}
& d(T 1, T 3)=1.1, d(1,3)=2.1, d(1, T 3)+d(T 1,3)=1 \\
& d(1, T 1) d(1, T 3)+d(3, T 3) d(3, T 1)=2.1, \max \{d(1, T 3), d(T 1,3)\}=1 .
\end{aligned}
$$

Case6. Let $(x, y) \in\{(2,3),(3,2)\}$, then

$$
\begin{aligned}
& d(T 2, T 3)=1.1, d(2,3)=1, d(2, T 3)+d(T 2,3)=2 \\
& d(2, T 2) d(2, T 3)+d(3, T 3) d(3, T 2)=3.1, \max \{d(2, T 3), d(T 2,3)\}=1
\end{aligned}
$$


In Case3, for all $\delta, \zeta \in[0,1)$ such that $\delta+\zeta<1$, we have

$$
\delta d(0,3)+\zeta \frac{d(0, T 0) d(0, T 3)+d(3, T 3) d(3, T 0)}{d(0, T 3)+d(3, T 0)}=\delta+\zeta<1<1.1=d(T 0, T 3) .
$$

This proves that $T$ does not satisfy in assumption of Theorem 11 of [1]. However in all cases, for $\lambda=\frac{69}{80}, \zeta=\frac{1}{8}$ and $\delta \in[0.57,1)$, we have

$$
d(T x, T y) \leq \lambda d(x, y)+\zeta \frac{d(x, T x) d(x, T y)+d(y, T y) d(y, T x)}{\max \{d(x, T y), d(y, T x)\}},
$$

and

$$
d(T x, T y) \leq \delta \max \left\{d(x, y), \frac{d(x, T x) d(x, T y)+d(y, T y) d(y, T x)}{\max \{d(x, T y), d(y, T x)\}}\right\}
$$

So by Theorem 2.1 or Theorem 2.2, 0 is the unique fixed point of $T$.

\section{References}

[1] J. Ahmad, M. Arshad, C. Vetro, On a Theorem of Khan in a Generalized Metric Space. Int. J. Anal., 2013 (2013), 6 pages. 1, 1, 2.3, 2.4

[2] A. Branciari, A fixed point theorem of Banach-Caccioppoli type on a class of generalized metric spaces, Publ. Math. Debrecen, 57 (2000), 31-37. 1.3, 1.4

[3] B. Fisher, On a theorem of Khan, Riv. Math. Univ. Parma., 4 (1978), 135-137. 1 , 1.1

[4] M. S. Khan, A fixed point theorem for metric spaces, Rend. Inst. Math. Univ. Trieste, 8 (1976), 69-72. 1

[5] B. Samet, Discussion on "a fixed point theorem of Banach-Caccioppoli type on a class of generalized metric spaces. Branciari," Publ. Math. Debrecen, 76 (2010), 493-494. 1

[6] I. R. Sarma, J. M. Rao, S. S. Rao, Contractions over generalized metric spaces. J. Nonlinear Sci. Appl. 2 (2009), 180-182. 1 